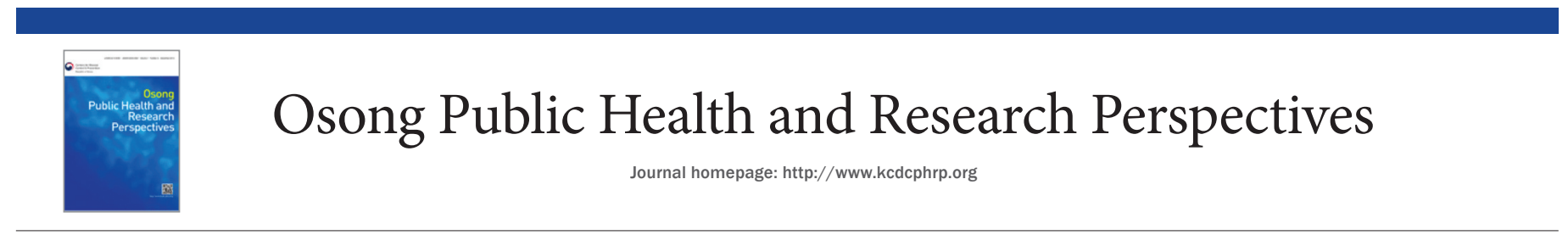

Editorial

\title{
One Bite Away
}

\author{
Hae-Wol Cho a,b,* \\ a Osong Public Health and Research Perspectives, Korea Centers for Disease Control and Prevention, Cheongju, Korea \\ ${ }^{b}$ College of Medicine, Eulji University, Daejeon, Korea
}

https://doi.org/10.24171/j.phrp.2019.10.6.01 pISSN 2210-9099 eISSN 2233-6052
Modern society is a borderless world. The global interconnectedness via travel, tourism, and international trade enables infectious diseases or pathogens to spread far and wide in unpredictable ways across the globe. To blockade the inflow of them preemptively, many countries are strengthening the nation's preparedness capacity of newly emerging infectious diseases using more vigilant surveillance and rapid risk communication. Dengue and zika virus infections are diseases endemic to the tropics, but pass over national boundaries.

Dengue fever is a condition caused by dengue virus [1]. Zika virus infection, is a mosquito-borne disease caused by the zika virus. Both these viral infections are transmitted to healthy humans by infected mosquitos, and from infected humans to mosquitos. Most cases of zika virus infections are asymptomatic, but when cases are symptomatic, they are usually mild and can resemble the symptoms of mild dengue fever [2]. Dengue and zika viral infections are transmitted to humans when an infected female Aedes mosquito (A. aegypti and $A$. albopitus) injects saliva into the host to prevent blood clotting, and enable feeding [3,4]. Infected mosquitos continue to transmit dengue or zika with each blood meal. When a mosquito bites an individual previously infected with dengue virus, they may transmit another serotype of dengue virus, which may increase the risk of severe complications such as dengue hemorrhagic fever [1]. In contrast, once humans are infected with zika virus, immune protection is conferred. Besides infection being transmitted via infected mosquitos, Zika virus is transmitted from mother to child, and may cause microcephaly and other severe brain defects in babies [4]. Furthermore, zika and dengue virus infections have been associated with Guillain-Barré syndrome, an autoimmune disorder attacking nerve cells and causing muscle weakness [5].

Dengue fever, and infection with zika virus are the 2 most common travel-related national notifiable diseases in the Republic of Korea. According to Korea Centers for Disease Control and Prevention, as of 2019, Korea has reported 150-300 cases of dengue virus infection and less than 10 cases of zika virus infections each year [6]. Most detected cases of dengue and zika virus infections were in Koreans who had traveled to Southeast Asia [7-11]. To conduct a feasible risk assessment of the potential threat of dengue and zika virus infections in Korean travelers, a model for incidence estimation is necessary.

In the current issue of Osong Public Health and Research Perspectives, a study by Chu et al [12] aimed to estimate the number of imported infections and the risk of infection, in Korean travelers visiting Southeast Asian and Latin American countries.

The authors analyzed the open dataset of visitors in 2016 and 2017 to 5 Southeast Asian countries (the Philippines, Indonesia, Thailand, Malaysia and Vietnam) who had dengue or zika virus infection. The authors calculated an estimate of the incidence of infection per year, and the average duration of travel (data from the World Tourism Organization). Estimated incidences of imported and laboratory-based confirmed dengue virus infections in 2016 were 7.46, 15.00, 2.14, 4.73 and 2.40 per 100,000 travelers visiting the Philippines, Indonesia, Thailand, Malaysia and Vietnam, respectively. Similarly, the incidence in 2017 were $2.55,1.65,1.53,1.86$ and 1.70 per 100,000 travelers, respectively.

For zika virus infections, the authors estimated that 60.1 infections with zika virus originated from Brazil in 2016, however, only 1 case of zika virus infection was reported to the

* Corresponding author: Hae-Wol Cho

College of Medicine, Eulji University, Daejeon, Korea

E-mail: hwcho47@gmail.com

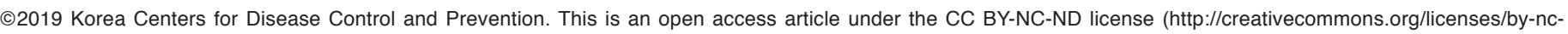
nd/4.0/). 
authorities. Similarly for the Maldives, in 2017 the estimated incidence of zika virus infections was 345.6 infections, but only 2 cases were notified to the Korean Authorities [12]. The authors concluded that due to the mild symptoms caused by dengue or zika virus infections, the actual number of infections may be under-reported. Therefore, the authors propose that appropriate risk assessment strategies of zika virus infections should be conducted using the calculated estimates. The under-reported actual incidences of infection may prevent the design of effective national strategies to contain the infection in the general population.

\section{References}

[1] World Health Organization [Internet]. Dengue and severe dengue. Geneva (Switzerland); World Health Organization: 2019. Available from: https:// www.who.int/en/news-room/fact-sheets/detail/dengue-and-severedengue.

[2] World Health Organization [Internet]. Zika virus. Geneva (Switzerland); World Health Organization: 2018. Available from: https://www.who.int/ en/news-room/fact-sheets/detail/zika-virus.

[3] Kularatne SA. Dengue fever. BMJ 2015;351:h4661.
[4] Chen LH, Hamer DH. Zika virus: Rapid spread in the Western hemisphere. Ann Intern Med 2016;164(9):613-5.

[5] European Centre for Disease Prevention and Control [Internet]. Zika virus. 2015. Available from: https://www.ecdc.europa.eu/en/zika-virus-disease.

[6] Korea Centers for Disease Control and Prevention. Public Health Wkly Rep 2019;12(39);1579-80. [in Korean].

[7] Korea Centers for Disease Control and Prevention [Internet]. Be wary of Zika infection during traveling Southeast Asia. Cheongju (Korea); Korea Centers for Disease Control and Prevention: 2018. Available from: https:// www.cdc.go.kr/board.es? mid=a20501000000\&bid=0015. [in Korean].

[8] Korea Centers for Disease Control and Prevention [Internet]. Be wary of mosquito-borne disease to travelers. Cheongju (Korea); Korea Centers for Disease Control and Prevention: 2018. Available from: https://www.cdc. go.kr/board.es? mid=a20501000000\&bid=0015. [in Korean].

[9] Korea Centers for Disease Control and Prevention [Internet]. Be wary of mosquitoes against imported dengue fever. Cheongju (Korea); Korea Centers for Disease Control and Prevention: 2016. Available from: https:// www.cdc.go.kr/board.es?mid=a20501000000\&bid=0015. [in Korean].

[10] Korea Centers for Disease Control and Prevention [Internet]. Dengue virus identified during mosquito monitoring. Cheongju (Korea); Korea Centers for Disease Control and Prevention: 2019. Available from: https://www. cdc.go.kr/board.es?mid=a20501000000\&bid=0015. [in Korean].

[11] Korea Centers for Disease Control and Prevention [Internet]. Be wary of Dengue fever during overseas travel. Cheongju (Korea); Korea Centers for Disease Control and Prevention: 2019. Available from: https://www.cdc. go.kr/board.es? mid=a20501000000\&bid=0015. [in Korean].

[12] Chu C, Shin ES. Estimation of the Size of Dengue and Zika Infection Among Korean Travelers to Southeast Asia and Latin America, 2016-2017. Osong Public Health Res Perspect 2019;10(6):394-8. 